\title{
Analysis of Dual Boundary Element in Shape Optimization
}

\author{
G. Gomes ${ }^{\mathrm{a}, *}$, A. Portela ${ }^{\mathrm{a}}$, B. Emidio ${ }^{\mathrm{a}}$, I. Lustosa ${ }^{\mathrm{b}}$ \\ ${ }^{a}$ UniversityofBrasilia, Campus Darcy Ribeiro, zip 70910-900, Brasilia/Brazil \\ ${ }^{b}$ Federal Institute of Technology and Science of Piau Campus Floriano, zip 64.800-000, Floriano/Brazil
}

\begin{abstract}
The numerical analysis with dual boundary element method for shape optimal design in twodimensional linear elastic structures is proposed. The design objective is to minimize the structural compliance, subject to an area constraint. Sensitivities of objective and constraint functions, derived by means of Lagrangean approach and the material derivative concept with an adjoint variable technique, are computed through analytical expressions that arise from optimality conditions. The dual boundary element method, used for the discretization of the state problem, applies the stress equation for collocation on the design boundary and the displacement equation for collocation on other boundaries. The perturbation field is described with linear continuous elements, in which the position of each node is defined by a design variable. Continuity along the design boundary is assured by forcing the end points of each discontinuous boundary element to be coincident with a design node. The optimization problem is solved by the modified method of feasible directions available in the PYOPT program and the accuracy and efficiency of the analysis is assessed through two examples of a plate with a hole, making this formulation ideal for the study of shape optimal design of structures.
\end{abstract}

Keywords:dual boundary element, shape optimization, feasible directions, pyopt software

\section{Introduction}

Shape optimal design is an important class of structural design problems in which the shape of a structural component is to be determined, subject to a given set of constraints. Sensitivity analysis plays a fundamental role in the determination of the shape optimal design. A unified theory of continuum shape design sensitivity analysis for elastic structures was developed by Choi et al. [1] and Haug et al. [2] by means of a variational formulation and material derivative concept. The major advantage of this theory is that the optimality conditions, derived on a continuum approach, lead to explicit design sensitivity expressions. The success of this formulation relies on the accurate computation of design sensitivities which, however, depend on boundary stresses that are often difficult to evaluate.

In general, numerical methods must be used for shape optimal design of engineering structures. The finite element method has a long and well- documented history in shape optimal design [3] to [6]. An intrinsic feature of the finite element method, common to all displacement based formulations, is the inaccuracy obtained in the computation of boundary stresses, as reported by Yang et al. [7] and Choi et al. [8]. ]. In order to overcome this difficulty, mixed formulations of the finite element method have been presented by MotaSoares et al. [9] and Rodrigues [10]. Another drawback of the finite element method is the need for continuously remeshing, in order to eliminate the error generated by mesh distortion during the shape redesign process, as reported by Rodrigues [10]. Despite these drawbacks, the finite element method has been the most widely used technique for shape optimal design.

The boundary element method (BEM) is a well-established numerical technique in the engineering community; see Brebbia et al. [11]. Its formulation in elastostatics can be based either on Betti's reciprocity theorem, Rizzo [12], or simply based on the classical work theorem, Portela [13]. The BEM has also been applied for shape optimal design, since it overcomes the difficultiespointed out for the finite element method, as reported by MotaSoares et al. [14]. Despite its good performance in shape optimal design, the standard BEM still lacks some accuracy in the evaluation of boundary stress, see MotaSoares et al. [15]. Thus, there is a clear need for an alternative modelling strategy for the analysis of shape optimal design of structures.

In this work the BEM is formulated by means of two independent boundary integral equations; the displacement and the stress boundary integral equations (DBEM). The use of the stress boundary integral equation, first reported by Portela et al. [16], allows high accuracy in the computation of boundary stresses, since this equation is independent of the displacement boundary integral equation and furthermore none approximation is introduced in its derivation.

The present paper is concerned with the application of the DBEM to the analysis of shape optimal design of two-dimensional linear elastic structures. The optimization problem is presented, the boundary integral equations are de- fined and the modelling strategy is discussed. Determining the optimal shape of the design boundary, an iterative analysis is performed using the Modified Method of Feasible Directions (MMFD) 
available in the pyOpt program [17]. In each iteration of the analysis, in which along the design boundary the state problem is modeled with straight quadratic boundary elements and the perturbation field is described with linear elements, the DBEM is applied for the evaluation of the objective and constraint functions, as well as their respective gradients. The effective treatment of the improper integrals of the boundary integral equations is a matter of fundamental importance in the DBEM. For curved boundary elements, the natural definition of ordinary finite-part integrals is applied to regularize the improper integrals. For straight boundary elements, analytic integration is carried out.

Finally, numerical results are obtained for a plate with a hole, for different loading conditions, and for the graphical visualization are used the MATLAB tool. It is demonstrated that the present modelling strategy can be used for shape optimal design of structures accurately and efficiently.

\section{Shape Optimal Design}

The design objective is to find the shape of a traction-free regular boundary such that, under an area constraint, the structure exhibits minimum compliance. Let $\Omega$ be the domain of a two-dimensional linear elastic structure and $\Gamma$ its boundary subdivided in $\Gamma_{\mathrm{u}}$ and $\Gamma_{\mathrm{t}}$. Consider that the structure has constrained displacements, defined on $\Gamma_{\mathrm{u}}$ and is loaded by a system of tractions, applied on $\Gamma_{\mathrm{t}}$. The design boundary $\Gamma_{\mathrm{d}}$, assumed as regular, is defined on $\Gamma_{\mathrm{t}}$, as presentedin Figure 1.

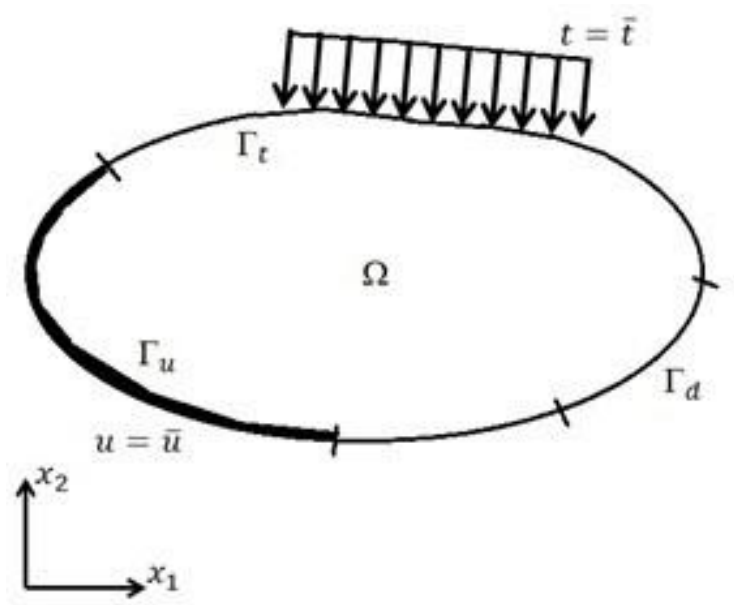

Figure1:Two-dimensionalelasticstructurewhere $\Omega$-domain; $\Gamma_{u}$-boundarywithfixeddisplacements $(\overline{\mathbf{u}}=\mathbf{0}) ; \Gamma_{t}$-boundarywithappliedtractions $; \Gamma_{d}$-designboundary $(\overline{\mathbf{t}}=\mathbf{0}) ; \mathbf{u}$-displacements; $\mathbf{t}$-tractions; $x_{i}-$ cartesiancoordinates.

The linear elastic problem is defined as

$G_{u_{j, i i}}+\frac{G}{1-2 v} u_{i, i j}=0$ in $\Omega$

withthe boundaryconditions

$\bar{u}=\bar{u}_{i}$ on $\Gamma_{u}$

and

$t_{i}=\bar{t}_{i}$ on $\Gamma_{t}$

where $u_{i}$ and $\quad t_{i}=\sigma_{i j} n_{j} \quad$ arerespectivelythedisplacementandthetractioncomponents; $\quad \sigma_{i j}$ arethestresscomponents; $n_{j}$ arethecomponentsoftheunitoutwardnormaltotheboundary; $G$

istheshearmodulusand $v$ isPoisson'scoefficient.Itisfurtherassumedthat isacinematicallyfixedboundary, thatis $\bar{u}_{i}=0$ on $\Gamma_{u}$ and $\Gamma_{d}$ isatraction-freeboundary, thatis $\bar{t}_{i}=0$ on $\Gamma_{d}$.

The objective function is the compliance of the structure, given by 


$$
\Psi_{0}\left(\Omega, u_{i}, t_{i}\left(\sigma_{i j}\right)\right)=\frac{1}{2} \int_{\Gamma} t_{i} u_{i} d \Gamma
$$

inwhich $u_{i}$ and $t_{i}$

arethestatevariables,obtainedfromthesolutionofthestateproblem,equations 1 to3.Thedesignoptimizationproble misexpressedasfinding $\Gamma_{d}$ suchthatthecompliance

isminimizedundertheconditionthatthetotalareaofthestructure $\Psi_{1}$

doesnotexceedaprescribedvalue $A$.Thisconstraintconditioncanbeexpressedas

$$
\Psi_{1}=\int_{\Omega} d \Omega=\frac{1}{2} \int_{\Gamma} x_{i} n_{i} d \Gamma \leq A,
$$

inwhich $x_{i}$ representcartesiancoordinates.Thenumericalsolutionofthisnon-

linearoptimizationproblem,derivedbymeansofLagrangeanapproachandthematerialderivativeconceptwithanadjoint variabletechnique,requirestheevaluationofthefirstvariationofboththeobjectiveandconstraintfunctionalfordesignsen sitivityanalysis. Thefirstvariationofboththecomplianceandtheareaconstraintisrespectivelygivenby

$$
\delta \Psi_{0}=-\int_{\Gamma_{d}} W v_{n} d \Gamma
$$

and

$$
\delta \Psi_{1}=\int_{\Gamma_{d}} v_{n} d \Gamma
$$

in which $W$ is the strain energy density and $v_{n}$ is the normal perturbation of the design boundary. Since $\Gamma_{d}$ is a traction-free boundary, the strain energy density can be defined as

$$
W=\frac{1}{2} \sigma_{I} \varepsilon_{I}=\frac{1}{2 E^{\prime}} \sigma_{I}^{2}=\frac{1}{2 E^{\prime}}\left(\sigma_{11}+\sigma_{22}\right)^{2}
$$

where $E^{\prime}=E /\left(1-v^{2}\right)$ inplanestrainand $E^{\prime}=E$ inplanestress,inwhich $E$ isthemodulusofelasticity; $\sigma_{I}$ istheprincipalstressand $\varepsilon_{I}$ isthecorrespondingprincipalstrain.

Thenormalperturbationofthedesignboundary $v_{n}$ isrepresentedinFigure2.Atapointofthedesignboundary $\Gamma_{d}$ ,adesignvariable b isdefinedasthenormofthepositionvectorofthatpointwithrespecttoapredefinedorigin $O$, asshowninFigure2.Thus,fora variationofthedesignvariable $\delta \mathbf{b}$

,thecorrespondingnormalperturbationofthedesignboundaryisgivenbytheorthogonalprojectionof $\delta \mathbf{b}$ ontothedirectionoftheunitoutwardnormaltotheboundary $\mathbf{n}$, thatis

$v_{n}=\delta \mathbf{b} . \mathbf{n}=\delta b_{i} n_{i}$

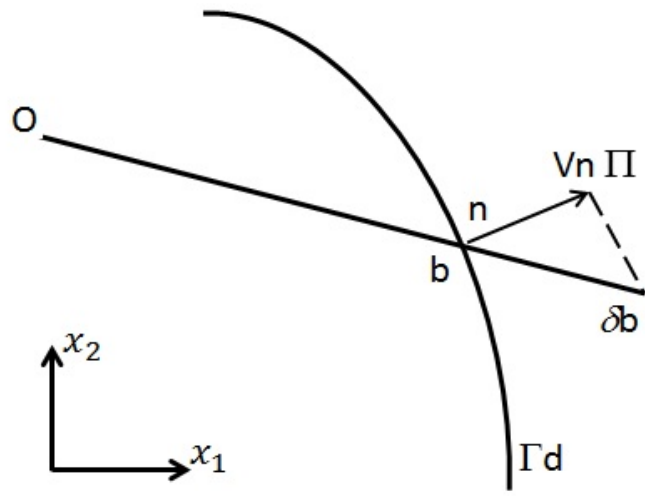

Figure 2: Normal perturbation of the design boundary where $b$ - design variable; $\delta b$ - variation of the design variable; $n$ - unit outward normal to the boundary; $v_{n}$ - normal perturbation of the design 
boundary; $x_{i}$ - cartesian coordinates; $O$ - origin of design variables.

\section{Boundary Integral Equations}

Theboundaryintegralequations,onwhichtheBEMisbased,arethedisplacementandthestressboundaryintegra lequations.ThepresentationoftheboundaryintegralequationsfollowsPortelaetal.[18].Intheabsenceofbodyforces, the boundaryintegralrepresentationofthedisplacementcomponents $u_{i}$, ataninternalpoint $\mathbf{X}^{\prime}$, isgivenby

$$
u_{i}\left(X^{\prime}\right)+\int_{\Gamma} T_{i j}\left(X^{\prime}, x\right) u_{j}(x) d \Gamma(x)=\int_{\Gamma} U_{i j}\left(X^{\prime}, x\right) t_{j}(x) d \Gamma(x)
$$

where $i$ and $j$ denote cartesian components; $T_{i j}\left(X^{\prime}, x\right)$ and $U_{i j}\left(X^{\prime}, x\right)$ represent the Kelvin traction and displacement fundamental solutions, respectively, at a boundary point $\mathbf{X}$. The distance between the points $\mathbf{X}^{\prime}$ and $\mathbf{X}$ is denoted by $r$. The integrals in equation (10) are regular, provided $r \neq 0$. As the internal point approaches the boundary, that is as $\mathbf{X}^{\prime} \rightarrow \mathbf{X}^{\prime}$, the distance $r$ tends to zero and, in the limit, the fundamental solutions exhibit singularities; they are a strong singularity of order $1 / r$ in $T_{i j}$ and a weak singularity of order $\ln (1 / r)$ in $U_{i j}$. Assuming continuity of the displacements at $\mathbf{x}^{\prime}$, this limiting process produces, in the first integral of equation (10), a jump term on the displacement components and an improper integral. For a boundary point, equation (10) can now be written as

$c_{i j}\left(x^{\prime}\right) u_{j}\left(x^{\prime}\right)+C V P \int_{\Gamma} T_{i j}\left(x^{\prime}, x\right) u_{j}(\mathrm{x}) \mathrm{d} \Gamma(\mathrm{x})=\int_{\Gamma} U_{i j}\left(x^{\prime}, x\right) t_{j}(\mathrm{x}) \mathrm{d} \Gamma(\mathrm{x})$

whereCVP stands for the Cauchy principal-value integral, and the coefficient $c_{i j}\left(x^{\prime}\right)$ is given by $\delta_{i j} / 2$ for a smooth boundary at the point $x$ ' $\left(\delta_{i j}\right.$ is the Kronecker delta).

Intheabsenceofbodyforces, thestresscomponents $\sigma i j$ areobtainedbydifferentiation ofequation(10), followedbytheapplication of Hooke'slaw; they aregivenby

$$
\sigma_{i j}\left(X^{\prime}\right)+\int_{\Gamma} S_{i j k}\left(X^{\prime}, x\right) u_{k}(x) d \Gamma(x)=\int_{\Gamma} D_{i j k}\left(X^{\prime}, x\right) t_{k}(x) d \Gamma(x)
$$

In this equation, $S_{i j k}\left(\mathrm{X}^{\prime}, \mathrm{x}\right)$ and $D_{i j k}\left(\mathrm{X}^{\prime}, \mathrm{x}\right)$ are linear combinations of derivatives of $T_{i j}\left(\mathrm{X}^{\prime}, \mathrm{x}\right)$ and $U_{i j}\left(\mathrm{X}^{\prime}, \mathrm{x}\right)$, respectively. The integrals in equation (12) are regular, provided $r \neq 0$. As the internal point approaches the boundary, that is $\mathrm{X}^{\prime} \rightarrow \mathrm{x}^{\prime}$, the distance $\mathrm{r}$ tends to zero and $S_{i j k}$ exhibits a hypersingularity of the order $1 / r^{2}$, while $D_{i j k}$ exhibits a strong singularity of the order $1 / r$. Assuming continuity of both strains and tractions at $x^{\prime}$, the limiting process produces improper integrals and jump terms in strains and tractions, in the first and second integrals of equation (12), respectively. For a point on a smooth boundary, these jump terms are equivalent to boundary stresses. Hence, equation (12) can now be written as

$$
\frac{1}{2} \sigma_{i j}\left(x^{\prime}\right)+\int_{\Gamma} S_{i j k}\left(x^{\prime}, x\right) u_{k}(x) d \Gamma(x)=H P V \int_{\Gamma} D_{i j k}\left(x^{\prime}, x\right) t_{k}(x) d \Gamma(x)
$$

whereHPVstands for the Hadamard [19] principal value integral. On a smooth boundary, the traction components, $\mathrm{t}_{\mathrm{j}}$, are given by

$$
\begin{aligned}
& \frac{1}{2} t_{j}\left(x^{\prime}\right)+n_{i}\left(x^{\prime}\right) C V P \int_{\Gamma} S_{i j k}\left(x^{\prime}, x\right) u_{k}(x) d \Gamma(x)= \\
& n_{i}\left(x^{\prime}\right) H V P \int_{\Gamma} D_{i j k}\left(x^{\prime}, x\right) t_{k}(x) d \Gamma(x)
\end{aligned}
$$

wherenidenotestheicomponentoftheunitoutwardnormaltothe boundary,atthepointx'.Equations(11)and(14)constitutethebasisoftheBEM.Onatractionfreeboundary,asinthecaseofthedesignboundary,theseequationsaresimplified;thedisplacementandthestressequation saregivenby 
$c_{i j}\left(x^{\prime}\right) u_{j}\left(x^{\prime}\right)+C V P \int_{\Gamma_{d}} T_{i j}\left(x^{\prime}, x\right) u_{j}(x) d \Gamma(x)=0$

and

$n_{i}\left(x^{\prime}\right)+H P V \int_{\Gamma_{d}} S_{i j k}\left(x^{\prime}, x\right) u_{k}(x) d \Gamma(x)=0$

respectively, where $\Gamma_{d}$ denotes the traction-free boundary.

BothCauchyandHadamardprincipal-

valueintegralsarefinitepartsofimproperintegrals,seeLinz[20]andPortela[21].Principal-

valueintegrals,computedthroughfinite-

partintegrals, wereintroducedintheboundaryelementmethodbyPortelaetal.[16].

\section{ModelingStrategy}

The necessary conditions for the existence of principal-value integrals, assumed inthederivationofthe boundary integralequations,imposespecialrestrictionsonthe boundarydiscretization.Considerthat boththegeometryand boundarystate bariablesaredescribed byacewisecontinuouslydifferentiableapproximation.Thus, CauchyandHadamardprincipal-

valueintegralsareequivalenttofinite-partintegralsoffirstandsecondorder,respectively.

Consider that collocation is always done at the boundary element nodes. Under this circumstance, the finitepart integral of first order, in the displacement equation (15), requires continuity of the displacement components at the nodes: any continuous or discontinuous boundary element satisfies this requirement. In the stress equation (16), the finite-part integral of second order requires continuity of the displacement derivatives at the nodes, on a smooth boundary: discontinuous quadratic boundary elements implicitly have the necessary smoothness, since the nodes are internal points of the element.

For the sake of efficiency and to keep the simplicity of the standard boundary elements, along the design boundary the present formulation uses discontinuous quadratic elements to model the elastic field and continuous linear elements to describe the perturbation field. The general modelling strategy developed in the present paper, schematically represented in Figure 3, can be summarized as follows:

- Discontinuous quadratic elements are used to model the design boundary, as well as other boundaries on which remeshing is allowed;

- All the remaining boundaries of the body are modeled with continuous quadratic elements;

- The stress equation (14) is applied for collocation at the nodes of the design boundary;

- The displacement equation (11) is applied for collocation at the nodes of the remaining boundaries;

- Continuous linear elements are used to model the normal perturbation field;

- On the design boundary, each quadratic element of the state problem is forced to be coincident with a linear perturbation element. Therefore, straight quadratic discontinuous elements are considered in this modelling strategy to describe the state problem on the design boundary, as shown in Figure 3.

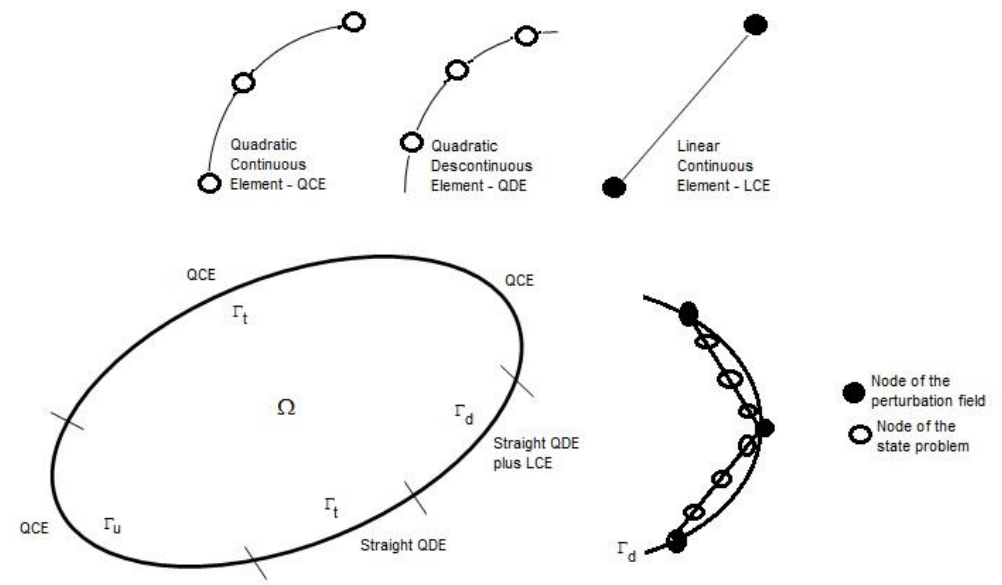

Figure 3: Modellingstrategy. 


\section{ComputationofGradients}

When the discretization of the design boundary, defined in the previous section, is considered, that is the use of straight discontinuous quadratic elements to model the elastic field and continuous linear elements to model the normal perturbation field, as represented in Figure 3, all the integrals in equations (6) and (7) are most effectively computed by direct analytic integration, which is presented in the following. Consider a straight discontinuous quadratic boundary element, with the nodes positioned arbitrarily at the points $\xi=-\frac{2}{3}, \xi=0$ and $\xi=+\frac{2}{3}$. The shape functionsof thiselement are given by:

$$
N_{1}=\xi\left(\frac{9}{8} \xi-\frac{3}{4}\right) \quad N_{2}=\left(1-\frac{3}{2} \xi\right)\left(1+\frac{3}{2} \xi\right) \quad N_{3}=\xi\left(\frac{9}{8} \xi+\frac{3}{4}\right)
$$

Consideralsoacontinuouslinearboundaryelement, withthenodespositionedatthepoints $\xi=-1$ and $\xi=+1$.Forthisele ment,theshapefunctionsaregivenby:

$$
M_{1}=\frac{1}{2}(1-\xi) \quad M_{2}=\frac{1}{2}(1+\xi)
$$

For an element of the design boundary, the gradients of both the compliance and the area constraint, respectively equations (6) and (7), are represented by:

$$
\delta \Psi_{0}^{e}=-\int_{\Gamma_{e}} W v_{n} d \Gamma=-\frac{1}{2 E^{\prime}} W_{i}\left[\int_{-1}^{+1} N_{1}(\xi) M_{j}(\xi) J(\xi) d \xi\right] v_{n j}
$$

and

$$
\delta \Psi_{1}^{e}=\int_{\Gamma_{e}} v_{n} d \Gamma=\left[\int_{-1}^{+1} M_{j}(\xi) J(\xi) d \xi\right] v_{n j}
$$

where $W i$ and $v n j$ arenodalvariablesand $J(\xi)$ istheJacobianofthecoordinatetransformation. Sincetheseelementsar eassumedstraight, ${ }_{2}^{l}$ inwhichlrepresentstheelementlength.Thus,equations 17 and18canbeintegratedanalyticallytoleadto, respectively:

$$
\delta \Psi_{0}^{e}=-\frac{l}{32 E^{\prime}}\left[\left(5 v_{n 1}+v_{n 2}\right) W_{1}+2\left(v_{n 1}+v_{n 2}\right) W_{2}+\left(v_{n 1}+5 v_{n 2}\right) W_{3}\right]
$$

and

$$
\delta \Psi_{1}^{e}=\frac{l}{2}\left(v_{n 1}+v_{n 2}\right)
$$

The improper integrals that arise in the boundary integral equations are easily handled by the classical singularity-subtraction technique, Davis and Rabinowitz [22], which leads to the natural definition of ordinary finite-part integrals. In the vicinity of a collocation node the regular part of the integrand is expressed as a Taylor's expansion. If a sufficient number of terms of the expansion are subtracted from the regular part of the integrand and then added back, the singularity can be isolated. The original improper integral is thus transformed into the sum of a regular integral and an integral of the singular function. This latter integral is then evaluated analytically, while standard Gaussian quadrature is used for numerical integration of the regular integral. Portela [21] has shown that this procedure is general and applicable to any type of boundary element, in which the necessary conditions for the existence of the finite-part integrals are implicitly satisfied.

Inthispaperthenormalperturbationfieldismodeledwithapiecewiselinearapproximation,asshowninFigure3.Consequently,inordertobecompatiblewiththe assumedlinearapproximationof theperturbationfield,the designboundaryismodeledaspiecewisestraight,whentheelasticfieldisregarded. Therefore, forpiece-wisestraightboundaries,alltheintegrals resulting from equations(15)and(16)aremosteffectivelycarriedoutbydirectanalyticintegration, whichispresentedinAppendix. 


\section{PYOPT Softwarecharacteristics}

The pyOpt is an open-source software Python-based package for formulating and solving nonlinear constrained optimization problems.Different type of open-source and licensed optimizers that solve the general nonlinear optimization problem have been integrated into the package, but in this work has been used the MMFD optimizer, an extension of the method of feasible directions, which utilizes the direction-finding subproblem from the Method of Feasible Directions [23] to find a search direction but does not require the addition of a large number of slack variables associated with inequality constraints.

The operatingstructure of $p y O p t$ follows thestepsin solvingoptimization problems, found on [17]:

1. Optimization Problem Definition: Solving general constrained nonlinear optimization, that is

$\min f(x)$ w.r.t. $x$

s.t.g_j(x)=0,j=1, .., m_e

$g_{-} j(x)<=0, j=m_{-} e+1, \ldots, m$

$x \_i \_L<=x \_i<=x \_i \_U, i=1, \ldots, n$

where:

- $\mathrm{x}$ is the vector of design variables

- $\mathrm{f}(\mathrm{x})$ is a nonlinear function

- $\mathrm{g}(\mathrm{x})$ is a linear or nonlinear function

- $\mathrm{n}$ is the number of design variables

- m_e is the number of equality constraints

- $m$ is the total number of constraints (number of equality constraints: $m \_i=m-m \_e$ )

2. Optimization Class: Instantiatethe optimization problem with the following settings

- Objective Function Template

- $\quad$ Objective value (f)

- Array of constraint values $(\mathrm{g})$

- Design Variables (continuous, integer and discrete)

- $\quad$ Single Design Variable

- A Group of Design Variables

- Constraints (inequality and equality)

- $\quad$ Single Constraint

- A Group of Constraints

3. Optimizer Class: Instantiate an Optimizer (e.g. CONMIM, MMFD). Here, we have been used the MMFD algorithm.

4. Optimizing:

- Solving the Optimization Problem (opt method)

- Output (initial values, specific solution and file output)

- History (stores all function evaluations)

5. Parallel Processing

- Gradient-based optimizers

- Parallel Objective Analysis (default)

- Parallel Gradients

- Population-based

- Parallel Objective Analysis

- Static Process Management

- Dynamic Process Management

1.1. Modelling the optimization problem

The two examplesof a plate, consideredin the next section,followingthe stepsgiven above, and the step

5 was based on thegradientoptimizer. Thus, the optimization problemwith pyOpt package consists of twosteps:

1. Setting up

- Set an Objective Function: in this case, the objective function is the compliance of the plate given by equation (4); 
- The objective function takes in the design variables array and returns a value. The array, in this case, it has five and four design variables, respectively for each example;

- Set an array of Constraints: in this case, the objective function is subject to an area constraint given by equation (5), in which the maximum admissible area is equal to the initial area of the plate;

- Initializes the optimization problem: opt_prob = pyOpt.Optimization('Optimization Problem with MMFD', objfunc);

- Finally, we complete the setupby adding, separately, the objective function, the designvariablesand the array of constraints:

- opt_prob.addObj('f') : add objective function name

- $\quad$ opt_prob.addVar('dv name','dv type',lower bound,upper bound,dv value) : add one-by-one design variables $(\mathrm{dv})$

- $\quad$ opt_prob.addConGroup('g name constr',total constr, 'type constr') : add a group of constraints

2. Solver

- Initializes the optimizer: in this case, we have used the MMFD (Modified Method of Feasible Directions) algorthm, that is

- $\quad m m f d=$ pyOpt.MMFD( )

- Define the Parallel Processing: in this case, the parallel-gradient has been used because the sensitivities are provided by the user. Here, the sensitivities are given by equations (19) and (20), respectively.

- $\quad m m f d\left(o p t \_p r o b\right.$, sens_type=gradfunc)

\section{NumericalResults}

Two applications of shape optimal design will be studied in this section. Consider an infinite square plate of side $\mathrm{h}$ with a central traction-free square hole of side a, loaded with biaxial uniform traction, $\bar{t} 1$ and $\bar{t} 2$, as presented in Figure 4a. The objective function is the compliance of the plate, subject to an area constraint, in which the maximum admissible area is equal to the initial area of the plate. When the central hole is considered as the design boundary, the analytical solution of this problem is given by Banichuk [24]; the optimal shape of the hole is a circumference, when $\bar{t} 1=\bar{t} 2$, and it is an ellipse with a semi-axis ratio equal to the ratio of the applied tractions, when $\bar{t} 1 \neq \bar{t} 2$. It was considered in both applications with the ratio $\mathrm{a} / \mathrm{h}=0.125$, in plane stress with the elastic constants $\mathrm{E}=200 \mathrm{GPa}$ and $v=0.3$. Since the problem is symmetric, only $1 / 4$ of the plate was considered in the analysis and a mesh with 16 quadratic boundary elements was set up, in which 4 discontinuouselements were used along the design boundary, as presented in Figure 4.b.

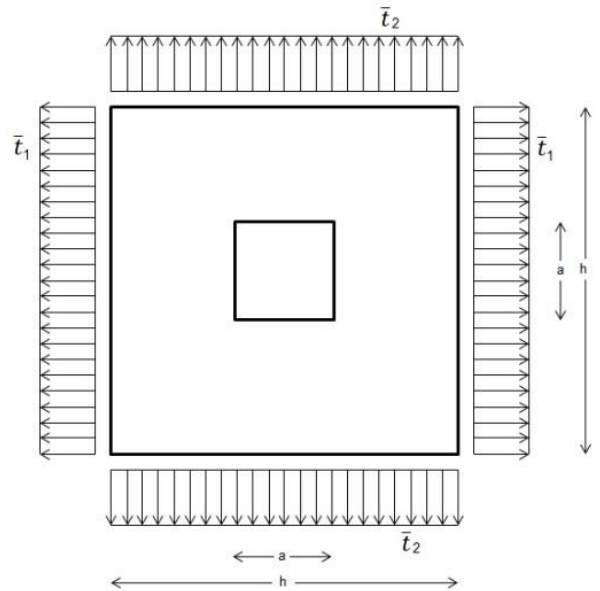

(a)

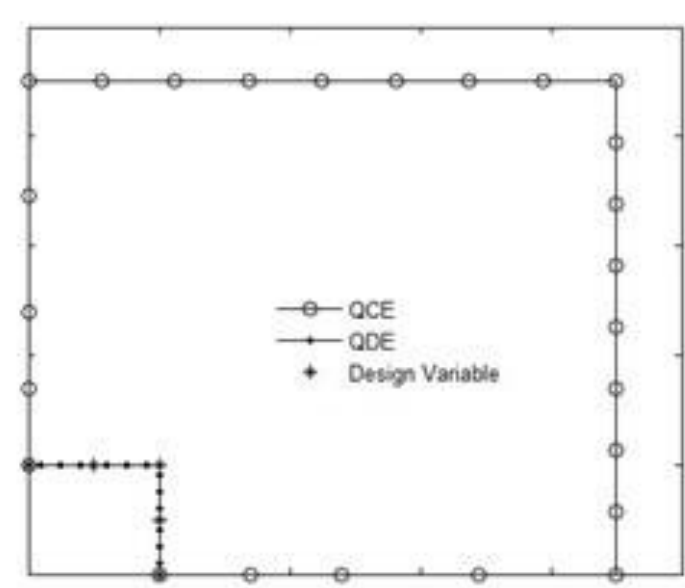

(b)

Figure 4: a) Infinite plate with a square hole $(a / h=0.125)$; b) Mesh with of $1 / 4$ the plate.

For the first application a loading ratio given by $\bar{t} 1 / \bar{t} 2=1$ was considered. Results of the shape optimal design problem were obtained with five design variables that are schematically represented in Figure 5. Figure6showstheinitialandfinalBEMmeshofthedesignboundary, which theanalyticalsolutionis a circumference.Here, the boundary design has been enlarged for better viewing. 


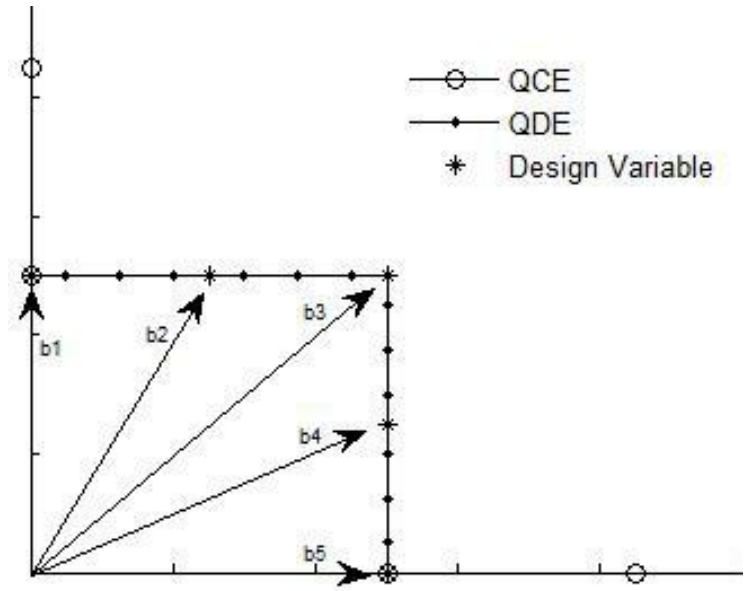

Figure 5: Design variables: b1 to b5 in the case $\bar{t}_{1} 1 / \bar{t}_{2}=1$; b1 to b4 in the case $\bar{t}^{-} 1 / \bar{t}_{2}=0,75$
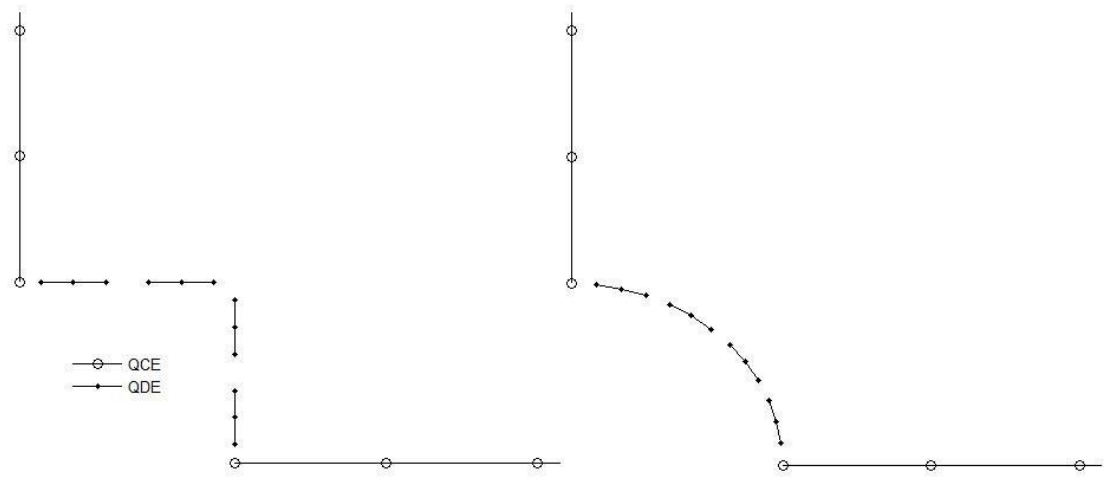

Figure 6: Initial and final BEM mesh of the design boundary $\left(\bar{t}_{1} 1 / \bar{t}_{2} 2=1\right)$

The design variables of the final shape with their respective values, which is the radius of the circumference, as well as the ratios between the mean value and each design variable, are presented in Table 1 . Figure 7 shows the radius and the normalized design variables, obtained with the pyopt program and compared with the reference radius, which we note that the maximum deviation from the mean value is $0.24 \%$.

Table 1: Radius and normalized designvariables

\begin{tabular}{|l|c|c|c|c|}
\hline \multirow{2}{*}{ DesignVariable } & \multicolumn{2}{|c|}{$\overline{T_{1} / \bar{t}_{2}}=1.0$} & \multicolumn{2}{c|}{$\bar{t}_{1} / \bar{t}_{2}=0.75$} \\
\cline { 2 - 5 } & Radius & $\Sigma \mathrm{b}_{\mathrm{j}} / 5_{\mathrm{j}}$ & Radius & $a / 2 b_{i}$ \\
\hline b1 & 0.501002 & 0.997406 & 0.336022 & 0.744000 \\
\hline b2 & 0.499002 & 1.001404 & 0.307882 & 0.812000 \\
\hline b3 & 0.498504 & 1.002403 & 0.281215 & 0.889000 \\
\hline b4 & 0.499002 & 1.001404 & 0.256148 & 0.976000 \\
\hline b5 & 0.501002 & 0.997406 & - & \\
\hline
\end{tabular}



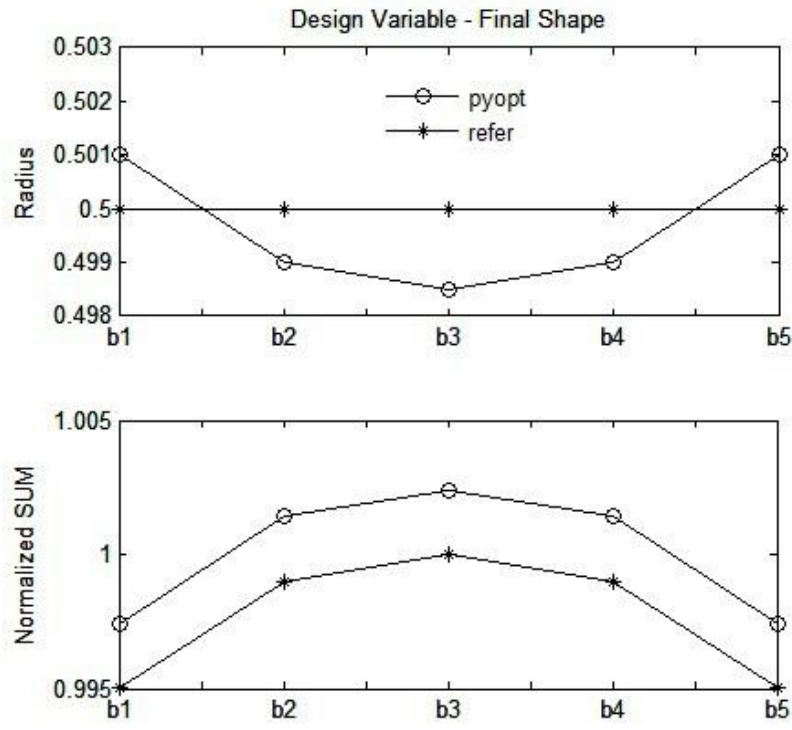

Figure 7: Radius and normalized design variables $\left(\mathrm{t}^{-} 1 / \mathrm{t}^{-} 2=1\right)$

The evolution of the design boundary during the optimization process is represented in Figure 8. Figure 9 shows the evolution of the compliance, normalized by its initial value, as well as the percentage error of the principal stresses, computed at BEM nodes of the design boundary. It can be seen that the error is slightly higher at the mid-side node of each element than it is at the end nodes. This is due to the straightness of BEM quadratic elements assumed along the design boundary, in order to be compatible with the linearapproximation denied for the perturbation field.

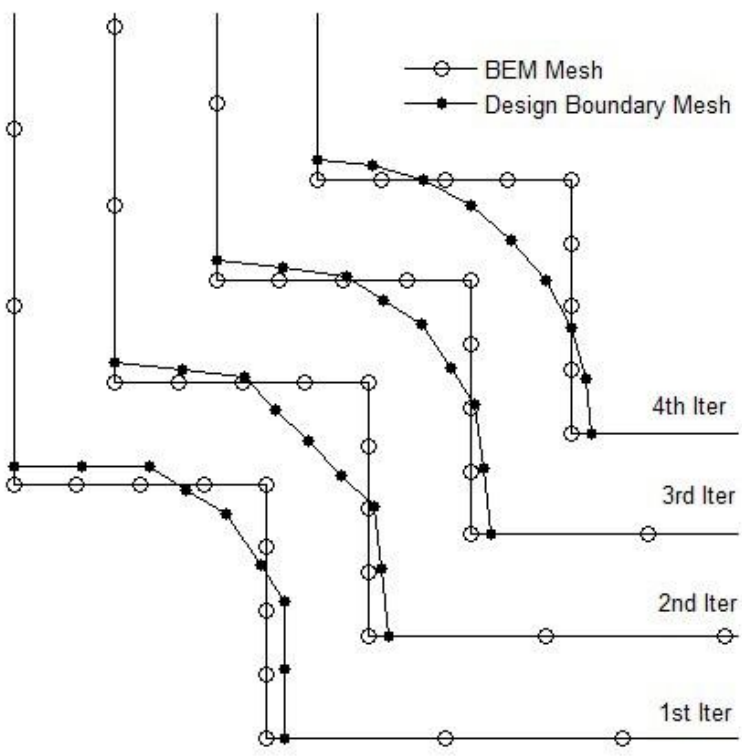

Figure 8: Evolution of the design boundary $\left(\bar{t} 1 / t^{-} 2=1\right)$ 

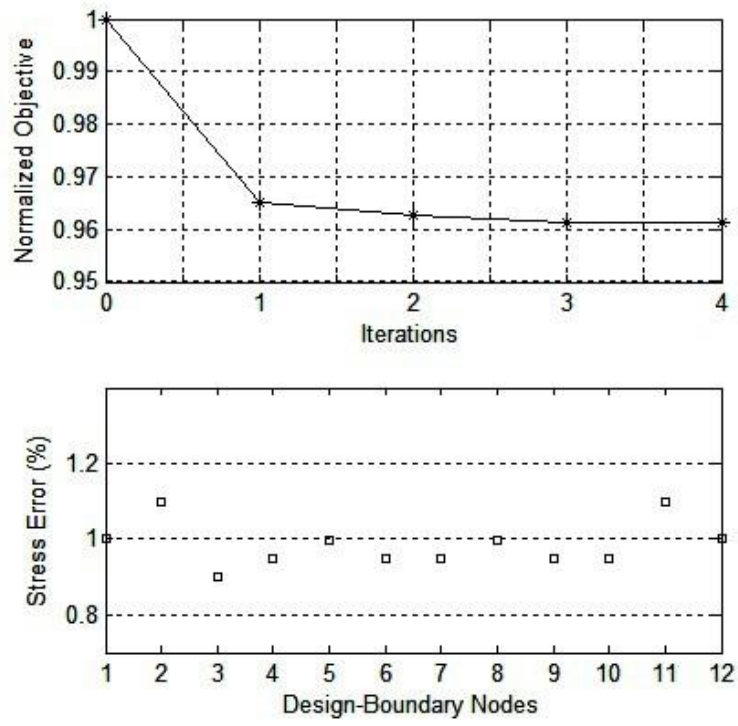

Figure 9: Evolution of the compliance and Error of principal stress at BEM nodes of the design boundary $\left(t^{-} 1 / t^{-} 2=1\right)$

A loading ratio given by $\left(\tau 1 / \tau^{-} 2=0.75\right)$ was considered in the second application. Results of the shape optimal design problem were obtained for this case with four design variables, schematically represented in Figure 5. The analytical solution of this problem is an ellipse with a semi-axis ratio equal to the ratio between the applied tractions. Table 1 and Figure 10 show the ratios between the minor semi-axis of the ellipse $a / 2$, and each design variable $b i$. It can be seen that the error obtained is less than $0.8 \%$.
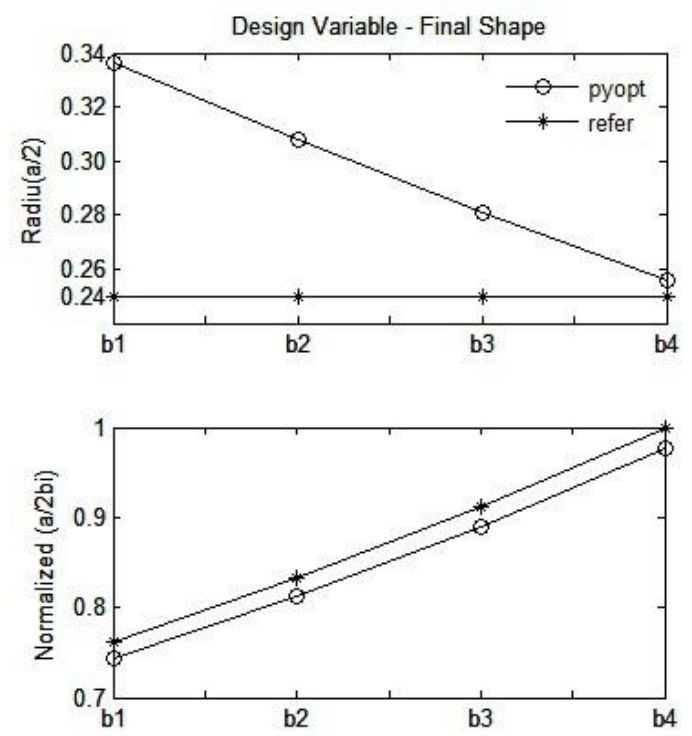

Figure 10:Radius andnormalized design variables $\left(\tau-1 / \tau^{-} 2=0.75\right)$

The evolution of the design boundary during the optimization process is represented in Figure 11. Figure 12 shows the evolution of the compliance, normalized by its initial value. The normalized compliance decreases monotonically until a minimum value is reached. This behavior is in contrast with the one present in the results reported by Leal et al. [25], obtained by a mixed formulation of the finite element method. 


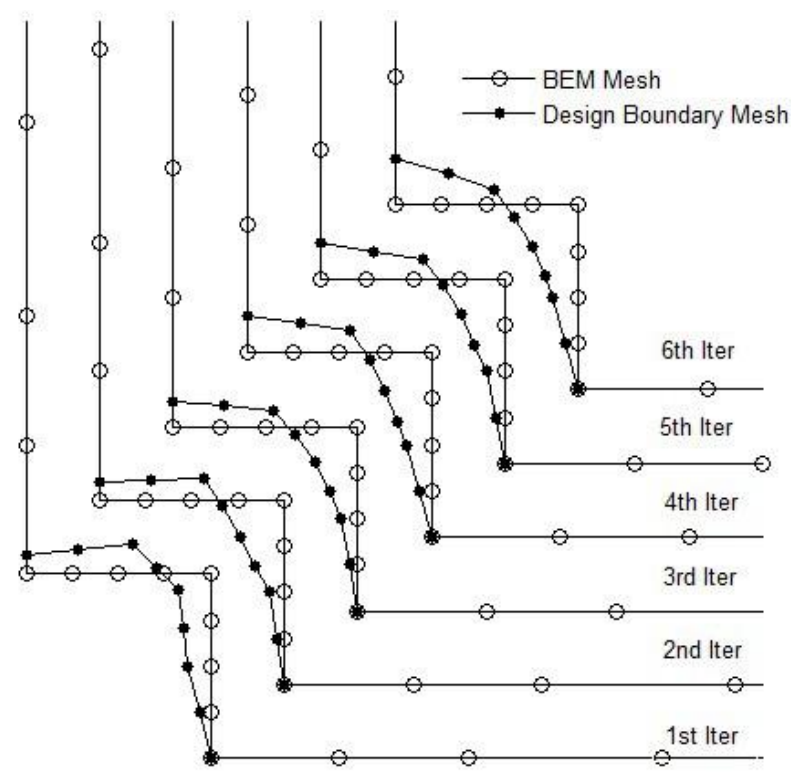

Figure 11: Evolution of the design boundary $\left(\bar{t}^{-} 1 / \bar{t}^{2}=0.75\right)$

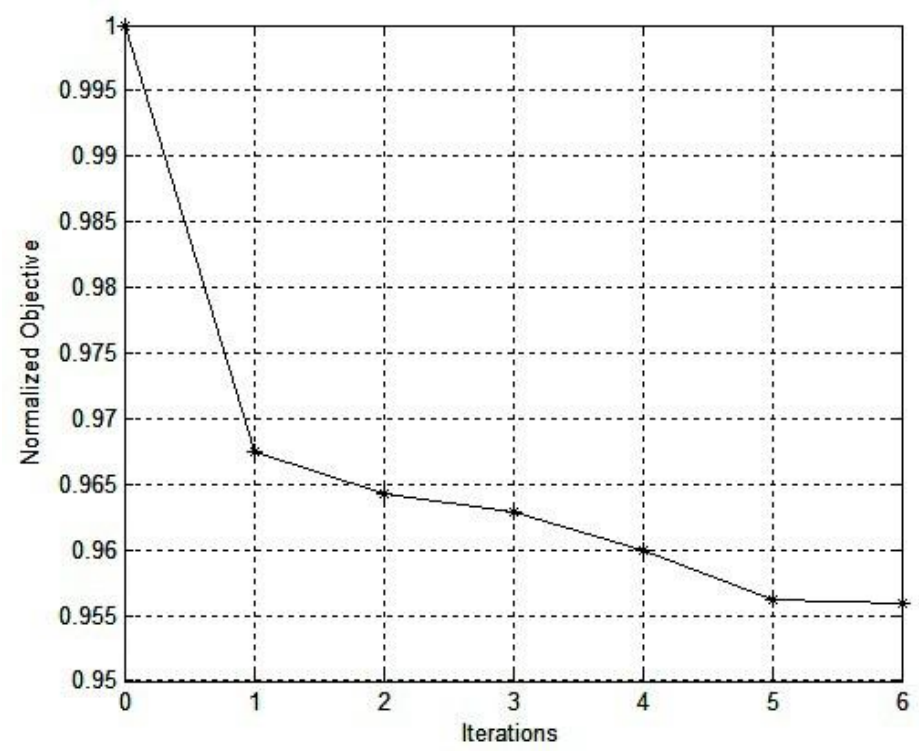

Figure 12: Evolution of the compliance $\left(\mathrm{t}^{-} 1 / \mathrm{t}^{-} 2=0.75\right)$

\section{FinalRemarks}

In this paper, the boundary element method is applied to the shape optimal design of two-dimensional linear elastic structures. The design objective is to minimize the structural compliance, subject to an area constraint. To solve this optimization problem, an iterative analysis is carried out using the modified method of feasible directions available in the program PYOPT. For each iteration of the analysis, in which along the design boundary the state problem is modeled with straight quadratic boundary elements and the perturbation field is described with linear elements, the dual boundary element method is applied for the evaluation of the objective and constraint functions, as well as their respective gradients. The reliability of the whole design strategy lies in the accuracy of the computation of boundary stresses.

The dual boundary element method incorporates two independent boundary integral equations: one is the stress boundary integral equation, used for collocation on the design boundary and the other is the displacement boundary integral equation, used for collocation on other boundaries. The use of the stress boundary integral equation, discretized with discontinuous quadratic elements to satisfy the necessary conditions for the existence of finite-part integrals, al- lows an efficient and accurate computation of stresses on the design boundary. This discretization strategy not only automatically satisfies the necessary conditions for the existence of the finite-part integrals, which occur naturally in the stress boundary integral equation, but also circumvents the problem of collocation at kinks and corners. This feature constitutes a practical advantage of the present formulation of the dual boundary element method over the finite element method and other boundary element formulations. 
Accurate results of shape optimal design were obtained for the problem of an infinite plate with a hole. The accuracy and efficiency of the implementation described herein make this formulation very effective for the study of shape optimal design of structures. Finally, it is important to highlight that the effectivity was also achieved with the use of the PYOPT program, since its object-oriented features has facilitated the communication and interaction between the formulations.

\section{Appendix}

Consider a discontinuous quadratic boundary element of general shape $\Gamma_{e}$ that contains the collocation node. The local parametric coordinate $\xi$ is defined, as usual, in the range $-1 \leq \xi \leq+1$ and the collocation node $\xi$ ' is mapped onto $\xi$ ' via the continuous element shape functions. The displacement components $u_{j}$ are approximated in the local coordinate by means of the nodal values $u_{j}^{n}$ and the discontinuous element shape functions. The first order finite-part integral of equation 15 can be expressed in the local coordinate as,

$$
C V P \int_{\Gamma_{e}} T_{i j}\left(x^{\prime}, x\right) u_{j}(x) d \Gamma(x)=u_{j}^{n} C V P \int_{-1}^{+1} \frac{f_{i j}^{n}(\xi)}{\xi-\xi^{\prime}} d \xi
$$

where $f_{i j}^{n}(\xi)$ is aregular function, given by the product of the fundamental solution, a shape function and the Jacobian of the coordinate transformation, multiplied by the term $\xi-\xi$ '. The integral of the right hand side of equation(21) can be transformed with the aid of the first term of a Taylor's expansion ofthe function $f_{i j}^{n}$, around the collocation node, to give

$$
C V P \int_{-1}^{+1} \frac{f_{i j}^{n}(\xi)}{\xi-\xi^{\prime}} d \xi=\int_{-1}^{+1} \frac{f_{i j}^{n}(\xi)-f_{i j}^{n}\left(\xi^{\prime}\right)}{\xi-\xi^{\prime}} d \xi+f_{i j}^{n}\left(\xi^{\prime}\right) C V P \int_{-1}^{+1} \frac{d \xi}{\xi-\xi^{\prime}}
$$

Now, the first integral of the right hand side is regular and the second one can be integrated analytically to give:

$$
C V P \int_{-1}^{+1} \frac{d \xi}{\xi-\xi^{\prime}}=\ln \left|\frac{1-\xi^{\prime}}{1+\xi^{\prime}}\right|
$$

In equation 22, the existence of the finite-part integral requires the Hldercontinuity of $f_{i j}^{n}$ at the collocation node. For the discontinuous element, this requirement is automatically satisfied because the nodes are internal points of theelement, where $f_{i j}^{n}$ is continuously differentiable. The second order finite-part integral of equation 16 can be expressed in the local parametric coordinate as

$$
H P V \int_{\Gamma_{e}} S_{i j k}\left(x^{\prime}, x\right) u_{k}(x) d \Gamma(x)=u_{k}^{n} H P V \int_{-1}^{+1} \frac{g_{i j k}^{n}(\xi)}{\left(\xi-\xi^{\prime}\right)^{2}} d \xi
$$

where $g_{i j k}^{n}(\xi)$ isaregularfunctionas $f_{i j}^{n}$ withtheJacobianmultipliedbytheterm $\left(\xi-\xi^{\prime}\right)^{2}$. The integral of the right hand side of equation 24 canbe transformed withtheaidofthefirstandsecondtermsofaTaylor'sexpansionofthedensity function $g_{i j k}^{n}$, in the neighborhood of the collocation node, to

$$
\begin{aligned}
& H P V \int_{-1}^{+1} \frac{g_{i j k}^{n}(\xi)}{\left(\xi-\xi^{\prime}\right)^{2}} d \xi \int_{-1}^{+1} \frac{g_{i j k}^{n}(\xi)-g_{i j k}^{n}\left(\xi^{\prime}\right)-g_{i j k}^{n(1)}\left(\xi^{\prime}\right)\left(\xi-\xi^{\prime}\right)}{\left(\xi-\xi^{\prime}\right)^{2}} d \xi+ \\
& g_{i j k}^{n}\left(\xi^{\prime}\right) H P V \int_{-1}^{+1} \frac{d \xi}{\left(\xi-\xi^{\prime}\right)^{2}}+g_{i j k}^{n(1)}\left(\xi^{\prime}\right) C P V \int_{-1}^{+1} \frac{d \xi}{\xi-\xi^{\prime}}
\end{aligned}
$$

where $g_{i j k}^{n(1)}$ denotes the first derivative of $g_{i j k}^{n}$. At the collocation node the function $g_{i j k}^{n}$ is required to have continuity of its second derivative or, at least, aHlder-continuous first derivative, for the finite-part integrals to exist. This requirementisalsoautomaticallysatisfiedbythediscontinuouselement,since the nodes are internal points of the element. Now, in equation 25, the first integraloftherighthandsideisregularandthethirdintegralisidenticalwith the one given in equation 23 . The second integral of the right hand side of equation 25 can be integrated analytically to give: 
$H P V \int_{-1}^{+1} \frac{d \xi}{\left(\xi-\xi^{\prime}\right)^{2}}=-\frac{1}{1+\xi^{\prime}}-\frac{1}{1-\xi^{\prime}}$

Equations22and25aretheordinarydouble-sidedfirstandsecondorderfinite-part integrals respectively, as defined by Kutt [23]. Inthispaperthenormalperturbationfieldismodeledwithapiece-wiselinear approximation,asshowninFigure 3. Consequently,inordertobecompatible with the assumed linear approximation of the perturbation field the design boundary is modeled as piece-wise straight, when the elastic field is regarded. For piece-wise straight boundaries, all the integrals in equations 22 and 25 are most effectively carriedoutbydirectanalyticintegration, whichispresentedin the following. Consider a straight discontinuous quadratic boundary element with nodes positioned at points $\xi=-2 / 3, \xi=0$ and $\xi=+2 / 3$ as done before. For this element, the integral of equation 15 is represented by:

$$
C P V \int_{\Gamma_{e}} T_{i j}\left(x^{\prime}, x\right) u_{j}(x) d \Gamma(x)=u_{j}^{n} C P V \int_{-1}^{+1} T_{i j}\left(\xi^{\prime}, x(\xi)\right) N_{n}(\xi) J(\xi) d \xi=\mathbf{h}_{i}^{n} \mathbf{u}^{n}
$$

where ${ }^{n}$ denotes the nodal displacement components and $J(\xi)$ is the Jacobian of the coordinate transformation. Because of the assumed straightness of the element, $J=l / 2$, where $l$ represents the element length and the matrix $\mathbf{h}^{n}$ is given by

$$
\mathbf{h}^{n}=\frac{1-2 v}{4 \pi(1-v)}\left[\begin{array}{cc}
0 & -1 \\
+1 & 0
\end{array}\right] C V P \int_{-1}^{+1} \frac{N_{n}}{\xi-\xi^{\prime}} d \xi
$$

The first order finite-part integrals are integrated analytically to give:

$$
\begin{aligned}
& C V P \int_{-1}^{+1} \frac{N_{1}}{\xi-\xi^{\prime}} d \xi=\frac{3}{4}\left(\frac{\xi^{\prime}\left(3 \xi^{\prime}-2\right)}{2} \ln \left|\frac{1-\xi^{\prime}}{1+\xi^{\prime}}\right|+3 \xi^{\prime}-2\right) \\
& C V P \int_{-1}^{+1} \frac{N_{2}}{\xi-\xi^{\prime}} d \xi=\frac{1}{2}\left(\frac{\left(3 \xi^{\prime}-2\right)\left(3 \xi^{\prime}+2\right)}{2} \ln \left|\frac{1+\xi^{\prime}}{1-\xi^{\prime}}\right|+9 \xi^{\prime}\right)
\end{aligned}
$$

and

$$
C V P \int_{-1}^{+1} \frac{N_{3}}{\xi-\xi^{\prime}} d \xi=\frac{3}{4}\left(\frac{\xi^{\prime}\left(3 \xi^{\prime}+2\right)}{2} \ln \left|\frac{1-\xi^{\prime}}{1+\xi^{\prime}}\right|+3 \xi^{\prime}+2\right)
$$

The integral of equation 16 is represented by

$$
H V P \int_{\Gamma_{e}} S_{i j k}\left(x^{\prime}, x\right) u_{k}(x) d \Gamma(x)=u_{k}^{n} H V P \int_{-1}^{+1} S_{i j k}\left(\xi^{\prime}, x(\xi)\right) N_{n}(\xi) J(\xi) d \xi=\overline{\mathbf{h}}_{i j}^{n} \mathbf{u}^{n}
$$

where $\overline{\mathbf{h}}^{n}$ the matrix is given by:

$$
\overline{\mathbf{h}}^{n}=\frac{E}{4 \pi\left(1-v^{2}\right)} \frac{2}{l} \mathbf{S}^{\prime} \operatorname{HVP} \int_{-1}^{+1} \frac{N_{n}}{\left(\xi-\xi^{\prime}\right)^{2}} d \xi
$$

The matrix $\mathbf{S}^{\prime}$, is given by

$$
\mathbf{S}^{\prime}=\left[\begin{array}{ll}
+n_{1}\left(2 n_{2}^{2}+1\right) & -n_{2}\left(-2 n_{2}^{2}+1\right) \\
+n_{1}\left(2 n_{1}^{2}-1\right) & -n_{2}\left(-2 n_{1}^{2}-1\right) \\
-n_{2}\left(2 n_{1}^{2}-1\right) & +n_{1}\left(-2 n_{2}^{2}+1\right)
\end{array}\right]
$$

where $n_{1}$ and $n_{2}$ are the components of the unit outward normal to the element. The second-order finite-part integrals of equation 33 are integrated analytically to give: 


$$
\begin{aligned}
& H P V \int_{-1}^{+1} \frac{N_{1}}{\left(\xi-\xi^{\prime}\right)^{2}} d \xi \frac{3}{4}\left(\left(3 \xi^{\prime}-1\right) \ln \left|\frac{1-\xi^{\prime}}{1+\xi^{\prime}}\right|+\frac{6 \xi^{\prime 2}-2 \xi^{\prime}-3}{\xi^{\prime 2}-1}\right) \\
& H P V \int_{-1}^{+1} \frac{N_{2}}{\left(\xi-\xi^{\prime}\right)^{2}} d \xi \frac{1}{2}\left(9 \xi^{\prime} \ln \left|\frac{1+\xi^{\prime}}{1-\xi^{\prime}}\right|-\frac{18 \xi^{\prime 2}-13}{\xi^{\prime 2}-1}\right)
\end{aligned}
$$

and

$$
H P V \int_{-1}^{+1} \frac{N_{3}}{\left(\xi-\xi^{\prime}\right)^{2}} d \xi \frac{3}{4}\left(\left(3 \xi^{\prime}+1\right) \ln \left|\frac{1-\xi^{\prime}}{1+\xi^{\prime}}\right|+\frac{6 \xi^{\prime 2}+2 \xi^{\prime}-3}{\xi^{\prime 2}-1}\right)
$$

Equation 33 shows that the terms of the matrix $\overline{\mathbf{h}}^{n}$ are inversely proportional to the element length $l$. This property is computationally advantageous because it can lead to diagonally dominant systems of algebraic equations.Higher accuracy will be obtained if the same order of approximation is used both for the perturbation field and BEM analysis, on the design boundary.

\section{References}

[1]. Choi, K.K., Haug, E.J., 1983, "Shape Design Sensitivity Analysis of Elastic structures”, J. Struct. Mech., 11, 231-269.

[2]. Haug, E.J., Choi, K.K, Komkov, V., 1986, "Design Sensitivity Analysis of Structural Systems", Academic Press, New York.

[3]. Chun, Y.W., Haug, E.J., 1978, “Two-dimensional Shape Optimal Design”,Int. J. Numer. Methods Eng., 13, $311-336$.

[4]. Choi, K.K., Haug, E.J., Hou, J.W., Sohoni, V.N., 1983, "Pshenichny'sLinearization Method for Mechanical System Optimization”, J. Mech. Des. ASME, 105, 97-104.

[5]. Haug, E.J., Choi, K.K., Hou, J.W., Yoo, Y.M., 1984, "A Variational Method for Shape Optimal Design of Elastic Structures", in New Directions in Optimal Structural Design, E. Atreket al. (Eds.), Wiley, New York, 1984, pp. $105-137$.

[6]. Kikuchi, N., Chung, K.Y., Torigaki, T., Taylor, J.E., 1986, “Adaptive Finite Element Methods for Shape Optimization of Linearly Elastic Structures”, Comp. Methods Appl. Mech. Eng., 57, 67-89.

[7]. Yang, R.J., Choi, K.K., 1985, “Accuracy of Finite Element Based Shape De- sign Sensitivity Analysis", J. Struct. Mech., 13, 223239.

[8]. Choi, K.K., Seong, H.W., 1986, “A Domain Method for Shape Design of Built-up Structures”, Comp. Methods Appl. Mech. Eng., $57,27-41$.

[9]. MotaSoares, C.A., Leal, R.P., 1987, "Mixed Elements in the Sensitivity Analysis of Structures”, Eng. Opt., 11, 227-237.

[10]. Rodrigues, H.C., 1988, "Shape Optimal Design of Elastic Bodies Using a Mixed Variational Formulation", Comput. Meth. Appl. Mech. Eng., 69, 29-44.

[11]. Brebbia, C.A., Dominguez, J., 1989, "Boundary Elements - an Introductory Course", Computational Mechanics Publications, Southampton, UK.

[12]. Rizzo, F. J., 1967, “An Integral Equation Approach to Boundary Value Problems of Classical Elastostatics”, Quarterly of Applied Mathematics, 25, 83-96.

[13]. Portela, A., 1980, "Theoretical Basis of Boundary Solutions for Linear Theory of Structures", in New Developments in Boundary Element Method, C.A.Brebbia (Ed.), Proc. of the Second International Seminar on Recent Advances in Boundary Element Methods, University of Southampton.

[14]. MotaSoares, C.A., Rodrigues, H.C., Choi, K.K., 1984, "Shape Optimal Structure Using Boundary Elements and Minimum Compliance Techniques", J. Mech. Trans. Automat. Des. ASME, 106, 518-523.

[15]. MotaSoares, C.A., Leal, R.P., Choi, K.K., 1987, "Boundary Elements in Shape Optimal Design of Structural Components", Computer Aided Optimal Design: Structural and Mechanical Systems, C.A. MotaSoares (Ed.), Springer-Verlag, Berlin.

[16]. Portela, A., Aliabadi, M.H., Rooke, D.P., 1992, "The Dual Boundary Element Method: Effective Implementation for Crack Problems", Int. J. Nu- mer. Methods Eng., 33, 1269-1287.

[17]. Perez R.E., Jansen P.W., and Martins J.R.R.A. (2012) pyOpt: A Python- Based Object-Oriented Framework for Nonlinear Constrained Optimization,Structures and Multidisciplinary Optimization, 45(1):101-118.

[18]. Portela, A., Aliabadi, M.H., Rooke, D.P., 1993, "Dual BoundaryElement Analysis of Linear Elastic Crack Problems", in Advanced Formulations in Boundary Element Methods, Computational Mechanics Publications, Southampton, U.K.

[19]. Hadamard, J., 1923, "Lectures on Cauchy's Problem in Linear Partial Differential Equations", New Haven, Yale University Press.

[20]. Linz, D.P., 1985, "On the Approximate Computation of Certain Strongly Singular Integrals", Computing, 35, 345-353.

[21]. Portela, A., 1992, "Dual Boundary Element Incremental Analysis of Crack Growth", Ph.D. thesis, Wessex Institute of Technology, Southampton, Portsmouth University, U. K..

[22]. Davis, P.J., Rabinowitz, P., 1984, "Methods of Numerical Integration", Academic Press, London.

[23]. Vanderplaats, G.N., 1987, “ADS - A Fortran Program for Automated Design Synthesis”, Version 2.01, Engineering Design Optimization.

[24]. Banichuk, N.V., 1983, "Problems and Methods of Optimal Structural Design", Plenum Press, New York.

[25]. Leal, R.P., MotaSoares, C.A., 1993, "Mixed Elements in Shape Optimal Design of Structures Based on Compliance", in Advanced Techniques in the Optimum Design of Structures, S. Hernandez (Ed.), Computational Mechanics Publications, Southampton. 